10

\title{
In-service monitoring techniques for cell transfer delay and cell delay variation in ATM networks
}

\author{
C. Roppel \\ Deutsche Telekom, Forschungs- und Technologiezentrum \\ P. O. Box 1000 03, D-64276 Darmstadt, Germany \\ Phone: +49615183-3817,e-mail: roppel@fz.telekom.de
}

\begin{abstract}
Cell transfer delay (CTD) and Cell delay variation (CDV) are ATM performance parameters. Monitoring CTD and CDV requires procedures for in-service measurement or estimation and for gaining the delay distribution statistics. This paper discusses the origin and definition of CTD and CDV, the latter being defined in terms of 2-point CDV and 1-point CDV. It outlines fundamental measurement techniques based on operation and maintenance (OAM) performance monitoring functions. Also, methods for estimating CTD and 2-point CDV are presented, which do not rely on the availability of synchronized clocks at the measurement points. They are based on a least-square regression analysis which estimates the time and frequency offset of the remote clock.
\end{abstract}

\section{Keywords}

ATM, cell transfer delay, cell delay variation, performance monitoring

\section{INTRODUCTION}

The great flexibility of ATM likewise involves high complexity. Controlling an ATM network proves to be a complicated and costly task. A major part of network management consists in monitoring the performance of the network, allowing appropriate action to be taken when failures or degradations in performance are detected. Operation and maintenance (OAM) in ATM forms an integral part of network management. In OAM, performance management fulfills the task of monitoring the normal functioning of network facilities. Performance management OAM cells provide a means for in-service monitoring of parameters related to network performance (Farkouh, 1993).

One generic performance parameter is information transfer speed (ITU-T, 1993a). In ATM, the parameters related to information transfer speed are cell transfer delay (CTD) and 
cell delay variation (CDV) (ITU-T, 1993b). Monitoring CTD and CDV requires procedures for measuring or estimating these parameters and for gaining statistics of the delay distribution.

The obvious way of measuring CTD is to transmit a time-stamped cell and to calculate the difference between the time the cell was received and the time the cell was sent. However, this method requires synchronized clocks at both the sending and receiving network elements. This paper describes a method for estimating CTD, which does not rely on the availability of synchronized clocks. CDV is split up into 2-point CDV and 1-point CDV. By means of the definition of CDV, estimation methods based on the use of time-stamped cells and on the analysis of the arrival times of user cells are described.

Section 2 discusses the definition and origin of CTD and CDV, and in Section 3 the basic concepts of OAM performance monitoring are described. Section 4 looks at measurement techniques for CTD, focussing on a method which does not require synchronized clocks. Methods for monitoring CDV are presented in Section 5, and Section 6 is intended to show the concepts outlined in the previous sections by means of an example.

\section{CELL TRANSFER DELAY AND CELL DELAY VARIATION}

Cell transfer delay (CTD) describes the delay a cell experiences during its transport through the network. The delay of cells of a virtual connection can vary from cell to cell. While CTD means the absolute delay of a cell, cell delay variation (CDV) is the variable part of CTD. From the viewpoint of quality of service it is important that the delay does not exceed a maximum, service-specific value, and that the maximum just as the minimum spacing between successive cells is kept inside certain boundaries. For multi-connection calls the allowed difference in mean delay of two virtual connections is limited.

CTD comprises several components (Cuthbert, 1993). These components are transmission delay, fixed switching delay and queueing delay. Transmission delay results from the finite signal propagation delay encountered on the physical medium. A typical value of a fiber-optic or a coaxial cable is approximately $5 \mu \mathrm{s} / \mathrm{km}$. Also part of this component is the delay introduced by transmission related equipment. The minimum time it takes a cell to pass through a switch is the fixed switching delay. It is composed of the time to perform a table look-up for header translation and routing and of the processing time in switching elements. For two $622.08 \mathrm{Mbit} / \mathrm{s}$ systems a minimum delay of $56.7 \mu \mathrm{s}$ and $64.8 \mu \mathrm{s}$ has been reported (Brinkmann, 1994). Queueing delay is the waiting time spent in cell queues. Queues are used for contention resolution in switches, for traffic shaping and for rate adaptation.

Other components of the overall end-to-end signal delay, which are segmention/ reassembly delay and coding/decoding delay, do not form part of CTD. Note that transmission delay, segmentation/reassembly delay and coding/decoding delay contribute the major part to the end-to-end signal delay.

While transmission delay and switching delay are fixed for a given connection, queueing delay is variable and depends on the loading of the network. Therefore, CTD has a fixed component $D_{\min }$, which is the minimum delay a cell experiences, and a variable component $D_{\text {var }}$, that is

$$
D=D_{\min }+D_{\text {var }} .
$$


CTD between two measurement points $\mathrm{MP}_{1}$ and $\mathrm{MP}_{2}$ is defined as the difference $D=t_{2}-t_{1}$ between the instant $t_{1}$, when the first bit of the ATM cell leaves measurement point $\mathrm{MP}_{1}$, and the instant $t_{2}$, when the last bit of the cell crosses measurement point $\mathrm{MP}_{2}$. It is $t_{2}>t_{1}$ and $t_{2}-t_{1} \leq T_{\max }$. For $t_{2}-t_{1}>T_{\max }$ the cell is considered to be lost (ITU-T, 1993b).

Concerning CDV, two parameters are defined: 2-point CDV and 1-point CDV (ITU-T, 1993b). 2-point CDV describes the variability of cell transfer delay introduced by the connection portion between measurement points $\mathrm{MP}_{1}$ and $\mathrm{MP}_{2}$. 1-point CDV describes the variability of cell arrival times at a measurement point with respect to the negotiated peak cell rate $1 / T_{P}$ (ITU-T, 1993c) of the monitored connection. It includes CDV characteristics of the cell source. 2-point CDV $v_{i}$ of cell $i$ is defined as the difference of the cell transfer delay $D_{i}$ of cell $i$ and the delay $D_{\text {ref }}$ of a reference cell between the two measurement points,

$v_{i}=D_{i}-D_{\text {ref }}$.

The distribution of 2-point CDV values is identical to the distribution of CTD values shifted by $D_{\text {ref }}$. 1-point CDV $y_{i}$ of cell $i$ is defined as the difference between the reference arrival time $c_{i}$ and the actual arrival time $t_{i}$,

$y_{i}=c_{i}-t_{i}$,

with the reference arrival time beeing defined in relation to the peak emission interval $T_{P}$,

$c_{0}=t_{0}, \quad c_{i+1}= \begin{cases}c_{i}+T_{P} & \text { for } y_{i} \geq 0, \\ t_{i}+T_{P} & \text { for } y_{i}<0 .\end{cases}$

This definition of the reference arrival time is insensitive to gaps in the cell stream due to lost cells. $y_{i} \geq 0$ corresponds to an early cell arrival, $y_{i}<0$ corresponds to a late arrival with respect to the reference pattern.

\section{OAM PERFORMANCE MONITORING}

While 1-point CDV can be monitored on the basis of cell arrival times only, monitoring CTD and, in general, also 2-point CDV requires the transmission of test cells containing time stamps. For in-service measurements, performance management operation and maintenance (OAM) cells may be used as test cells. They allow performance monitoring of segments and end-to-end connections of virtual path connnections (VPC) and virtual channel connections (VCC). The information flow is referred to as F4 flow at the VP level and as F5 flow at the VC level (ITU-T, 1993d).

VP level OAM cells have the same VPI as the user cells of that connection, but are identified by specific VCI values. Cells of a segment $\mathrm{F} 4$ flow have $\mathrm{VCI}=3$, and cells of an end-to-end F4 flow have VCI=4. VC level OAM cells have the same VPI/VCI as the user cells of that connection, but have specific payload type (PT) values. Cells of a segment F5 flow have PT=4, and cells of an end-to-end F5 flow have PT=5.

For delay measurements performance management OAM cells contain a 32 bit time stamp field. The delay the OAM cell experiences is considered as a random sample of the delay of 
user cells. Performance monitoring is activated and deactivated on demand between the two end-points of the connection or segment.

\section{ESTIMATING CTD}

Figure 1 shows a general arrangement for the measurement of CTD. Process $P_{1}$ of network element (NE) 1 initiates the measurement on an existing connection between $P_{1}$ and process $\mathrm{P}_{2}$ of NE 2. NE 1 has a local clock $C_{1}(t)$ and NE 2 has the local clock $C_{2}(t) . \mathrm{P}_{1}$ sends timestamped cells upstream on the predefined connection to $\mathrm{P}_{2}$, which experience a delay $D_{u}$ in the network. In the downstream direction, cells experience a delay $D_{d}$.

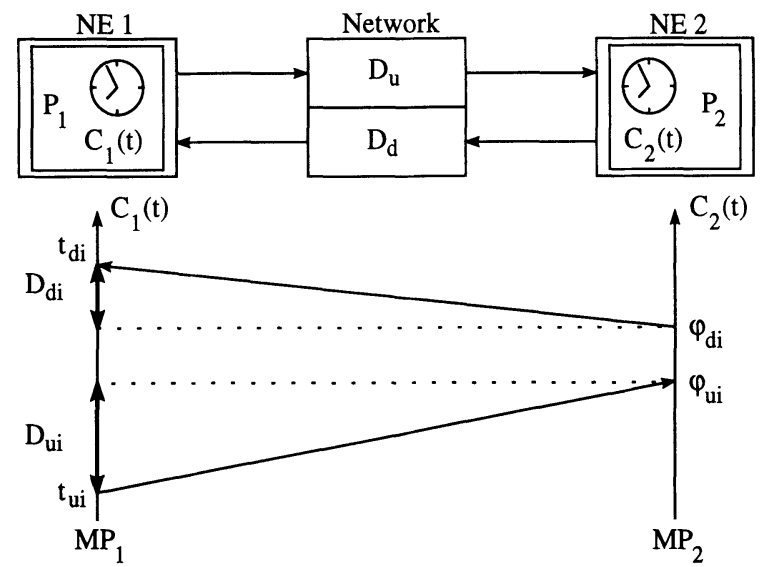

Figure 1 General configuration for CTD measurement.

If the clocks $C_{1}(t)$ and $C_{2}(t)$ are synchronized, then $C_{1}(t)=C_{2}(t)=t$. The upstream CTD of cell $i, D_{u i}$, can be calculated by subtracting the time $t_{u i}$ the cell was sent from the time $\varphi_{u i}$ it was received. Similarly, the downstream delay $D_{d i}$ is obtained by subtracting $\varphi_{d i}$ from $t_{d i}$. Synchronization of clocks at different locations with sufficient accuracy can be achieved by using synchronization signals of the satellite based global positioning system GPS. With the standard positioning service provided by GPS clocks can be synchronized to UTC (universal time coordinated) with an accuracy of about $100 \mathrm{~ns}$ (Klepczynski, 1983). However, this means additional cost and complexity at the network elements for equipment and maintenance, which would not be justifiable especially in the customer and access area.

A simple estimation of CTD can be based on the measurement of the round trip delay $t_{\mathrm{RTD}}$, where the time-stamped cells are looped back at the remote NE. Up- and downstream delay are estimated by $\hat{D}_{u i}=\hat{D}_{d i}=t_{\mathrm{RTD}} / 2$ (Gruber, 1993). However, this approach is only useful for estimating the mean CTD. It can not be used for evaluating the delay distribution.

If the clocks $C_{1}(t)$ and $C_{2}(t)$ are not synchronized, another approach is to estimate the offset and drift of the remote clock $C_{2}$ relative to $C_{1}$. If these parameters are known, it is possible to calculate the correct delays by scaling the time $\varphi$ of $C_{2}$ to the time of $C_{1}$. In order 
to estimate the parameters of the remote clock, a model of the clock is required, which is described in the following section.

\subsection{Model of a clock}

A clock consists of an oscillator and a counter incrementing with a specified number of cycles of the oscillator. Free running clocks show a time offset, a frequency offset and a frequency offset rate relative to each other, determined by the properties of the oscillator. This is modelled by the following equation:

$C(t)=t+\Delta T_{0}+\alpha\left(t-t_{0}\right)+\frac{\beta}{2}\left(t-t_{0}\right)^{2}+\varepsilon(t)$.

The error of a free running clock relative to a reference clock $C_{r}(t)=t$ is $\Delta T(t)=C(t)-t$. $\Delta T_{0}=\Delta T\left(t_{0}\right)$ is the time offset at time $t_{0}, \alpha=\Delta \dot{T}\left(t_{0}\right)=\left(f-f_{r}\right) / f_{r}$ is the frequency offset, and $\beta=\Delta \ddot{T}\left(t_{0}\right)=\left(\dot{f}-\dot{f}_{r}\right) / f_{r}$ is the frequency offset rate, where $f$ is the frequency of the oscillator of the local clock and $f_{r}$ is the frequency of the oscillator of the reference clock. While $\alpha$ and $\beta$ model the oscillator long term stability, i. e. systematic changes in frequency, $\varepsilon(t)$ models the oscillator short term stability, i. e. random frequency variations (Ellingson, 1973).

Usually, the frequency source will be a crystal device. Then, the frequency offset typically lies in the range of a few ppm. The frequency offset rate typically is better than $10^{-13} \mathrm{~s}^{-1}$. For measurement times up to a few minutes, the frequency offset rate may be neglected. Similarly, with reasonable intervals between individual measurements, the short term stability need not be considered.

The time between two increments of the counter is the resolution of the clock. This results in a quantization error. Assume that the measurement starts at time $t_{0}=0$. Then, if the two last terms in (5) are neglected, the clock can be modelled by

$$
C(t)=\operatorname{Int}\left(t+\Delta T_{0}+\alpha \cdot t\right)
$$

where the function $\operatorname{Int}(x)$ returns the integer part of $x$, i. e. Int $(x)=x-x \bmod 1$. This models the quantization error, where $t$ is given in units corresponding to the resolution of the clock (e. g. in units of one microsecond).

\subsection{Estimation of time and frequency offset of a remote clock}

For CTD measurement, the local clock $C_{1}$ at NE 1 is used as a reference clock, i. e. $C_{1}(t)=t$. Assuming a clock model as given by Equation (6), the time offset $\Delta T_{0}$ and the frequency offset $\alpha$ of the remote clock $C_{2}$ relative to $C_{1}$ can be estimated with a least-square linear regression analysis (Duda, 1987). This analysis is based on a sequence of time stamps obtained from a measurement as shown in Figure 1.

This supposes that all time stamps $t_{u i}, \varphi_{u i}, t_{d i}$ and $\varphi_{d i}$ are collected at one network element. An obvious way to achieve this is performing a loopback of the time-stamped cells at the remote NE, although this is not imperative. However, in current standards the loopback of performance management OAM cells for delay measurements is only discussed as a future 
option (ITU-T, 1993d). In the following, we assume that upon reception of a time-stamped cell, NE 2 inserts a receiving time stamp and immediately loops back the cell to NE 1 . It is assumed that the time for OAM cell processing can be neglected.

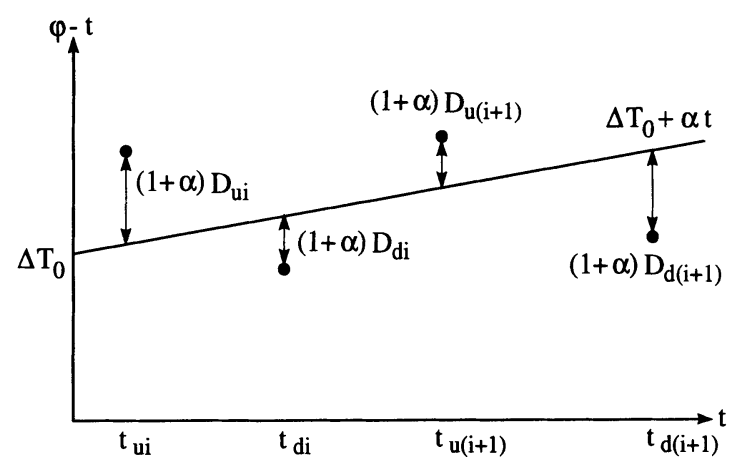

Figure 2 Data points on the $(t, \varphi-t)$ plane.

Upstream, process $P_{1}$ sends cells with time stamps $t_{u i}$ to process $P_{2}$, which receives them after $t_{u i}+D_{u i}$, inserts a time stamp $\varphi_{i}$ and sends them back to $P_{1}$. Here, the cells are received at time $t_{d i}=t_{u i}+D_{u i}+D_{d i}$. According to (6), neglecting the $\operatorname{Int}(x)$ function, $\varphi_{i}$ is given by

$\varphi_{i}=C_{2}\left(t_{u i}+D_{u i}\right)=\Delta T_{0}+(1+\alpha)\left(t_{u i}+D_{u i}\right)$.

Now consider the points $\left(t_{u i}, \varphi_{i}-t_{u i}\right)$ and $\left(t_{d i}, \varphi_{i}-t_{d i}\right)$ in the $(t, \varphi-t)$ plane, their y-coordinates being

$\varphi_{i}-t_{u i}=\Delta T_{0}+\alpha \cdot t_{u i}+(1+\alpha) D_{u i}$,

$\varphi_{i}-t_{d i}=\Delta T_{0}+\alpha \cdot t_{d i}-(1+\alpha) D_{d i}$.

The points $\left(t_{u i}, \varphi_{i}-t_{u i}\right)$ lie by $(1+\alpha) D_{u i}$ above the straight line represented by $\Delta T_{0}+\alpha \cdot t$ as illustrated in Figure 2. Similarly, the points $\left(t_{d i}, \varphi_{i}-t_{d i}\right)$ lie by $(1+\alpha) D_{d i}$ below this line.

The delays $D_{u i}$ and $D_{d i}$ are random variables. Given a sufficient number of data points, we can estimate the parameters of the remote clock $C_{2}$ by means of a least-square linear regression analysis. The parameters of the regression curve are fitted such that the sum of squares of the vertical spacings of all points to the curve is at a minimum. Sending $n$ cells results in $2 n$ data points, the estimate $\hat{\alpha}$ being

$$
\hat{\alpha}=\frac{\frac{1}{2 n}\left(\sum_{i=1}^{n} t_{u i} \cdot\left(\varphi_{i}-t_{u i}\right)+\sum_{i=1}^{n} t_{d i} \cdot\left(\varphi_{i}-t_{d i}\right)\right)-\bar{t} \cdot \overline{\varphi-t}}{\frac{1}{2 n}\left(\sum_{i=1}^{n} t_{u i}^{2}+\sum_{i=1}^{n} t_{d i}^{2}\right)-\bar{t}^{2}},
$$

where $\bar{t}$ and $\overline{\varphi-t}$ are given by 
$\bar{t}=\frac{1}{2 n}\left(\sum_{i=1}^{n} t_{u i}+\sum_{i=1}^{n} t_{d i}\right), \overline{\varphi-t}=\frac{1}{2 n}\left(\sum_{i=1}^{n}\left(\varphi_{i}-t_{u i}\right)+\sum_{i=1}^{n}\left(\varphi_{i}-t_{d i}\right)\right)$

Linear regression can also give an estimate of the time offset $\Delta T_{0}$ based on all data points with $\Delta \hat{T}_{0}=\overline{\varphi-t}-\hat{\alpha} \cdot \bar{t}$. But this will yield an estimate close to the actual value only if the delay distribution has a zero mean, or $\bar{D}_{u}-\bar{D}_{d}=0$. In other words, the mean delay in both the up- and downstream direction must be equal.

A better estimate for $\Delta T_{0}$ can be obtained by observing that the delay has a fixed, minimum component $D_{\min }$ (Equation (1)). Since cells of a virtual connection are routed on the same physical links for both the up- and downstream direction, the fixed delay $D_{\min }$ is assumed to be equal for both directions. Given a large number of data points, some of them will show a delay equal or close to $D_{\min }$. Therefore, an estimate $\Delta \hat{T}_{0}$ for the time offset can be obtained as follows:

1. Select $m$ data points with the minimum upstream delay and $m$ points with the minimum downstream delay. To determine the points with the minimum delay, the time stamps $\varphi_{i}$ of $C_{2}$ have to be corrected for the frequency offset $\alpha$. The points for the up- and downstream direction are then given by

$$
\operatorname{Min}\left\{\frac{\varphi_{i}}{1+\hat{\alpha}}-t_{u i}\right\}, \operatorname{Max}\left\{\frac{\varphi_{i}}{1+\hat{\alpha}}-t_{d i}\right\}
$$

respectively.

2. Calculate $\Delta \hat{T}_{0}$ using the $2 m$ data points. $\Delta \hat{T}_{0}$ in general is given by

$$
\Delta \hat{T}_{0}=\overline{\varphi-t}-\hat{\alpha} \cdot \bar{t}=(1+\hat{\alpha}) \cdot\left(\frac{\bar{\varphi}}{(1+\hat{\alpha})}-\bar{t}\right)
$$

Using Equation (11) we have

$$
\Delta \hat{T}_{0}=(1+\hat{\alpha}) \cdot \frac{1}{2}\left(\frac{1}{m} \sum_{j=1}^{m} \frac{\varphi_{j}}{1+\hat{\alpha}}-t_{u j}+\frac{1}{m} \sum_{j=1}^{m} \frac{\varphi_{j}}{1+\hat{\alpha}}-t_{d j}\right) .
$$

The procedure of estimating $\alpha$ and $\Delta T_{0}$ can be summarized as follows. First, $\hat{\alpha}$ is calculated by means of Equation (9) using all $2 n$ data points. Next, we select $2 m$ points with the minimum delay for the up- and downstream direction, respectively, as given by Equation (11). To do this, we need to know $\hat{\alpha}$. Now $\Delta \hat{T}_{0}$ is calculated using $\hat{\alpha}$ and the data points with minimum delay (Equation (13)). In principle, $m=1$ would be sufficient for the determination of $\Delta \hat{T}_{0}$, if these two points did correspond exactly to the minimum delay $D_{\min }$. In practice, however, $m>1$ would be selected in order to determine $\Delta \hat{T}_{0}$ from the average of the points corresponding to a delay close to $D_{\min }$. 


\subsection{Calculation of the delay}

When we know the estimates $\Delta \hat{T}_{0}$ and $\hat{\alpha}$ for the time and frequency offset of the clock $C_{2}$ relative to $C_{1}$, we can scale the time $\varphi$ of $C_{2}$ to the time of $C_{1}$ :

$t=\frac{\varphi-\Delta \hat{T}_{0}}{1+\hat{\alpha}}$

The delay is now obtained by forming the difference between the time stamps of $C_{1}$ and the scaled time stamps of $C_{2}$. The estimated values for the up- and downstream delay, $\hat{D}_{u i}$ and $\hat{D}_{d i}$, are:

$$
\hat{D}_{u i}=\frac{\varphi_{i}-\Delta \hat{T}_{0}}{1+\hat{\alpha}}-t_{u i}, \quad \hat{D}_{d i}=t_{d i}-\frac{\varphi_{i}-\Delta \hat{T}_{0}}{1+\hat{\alpha}} .
$$

\section{ESTIMATING CDV}

A general configuration for the measurement of CDV is shown in Figure 3. Since CDV is estimated by the receiving network element at $\mathrm{MP}_{2}$, the clock $C_{2}$ of this $\mathrm{NE}$ is used as a reference, and the time stamps of $C_{2}$ are denoted by $t_{i}$. For $C_{1}$ the considerations of Section 4.1 apply.

For the measurement of 2-point $\mathrm{CDV}$, the $\mathrm{NE}$ at $\mathrm{MP}_{1}$ inserts a time stamp $\varphi_{i}$ in a test cell and sends it to the NE at $\mathrm{MP}_{2}$. The cell experiences a delay $D_{i}$ and arrives at time $t_{i}$ at $\mathrm{MP}_{2}$. The time stamps $\varphi_{i}$ are given by

$\varphi_{i}=C_{1}\left(t_{i}-D_{i}\right)=\Delta T_{0}+(1+\alpha)\left(t_{i}-D_{i}\right)$.

While a time offset $\Delta T_{0}$ cancels out in the calculation of $\hat{v}_{i}=\hat{D}_{i}-\hat{D}_{\text {ref }}$ (Equation (2)), a frequency offset $\alpha$ has to be estimated in order to determine the correct $v_{i}$. This is done with a least-square regression analysis as described in Section 4.2 using the points $\left(t_{i}, \varphi_{i}-t_{i}\right)$. $\hat{D}_{i}$ and $\hat{D}_{\text {ref }}$ are then given by the difference between the time stamps $t_{i}$ and $\varphi_{i}$, with $\varphi_{i}$ scaled by $1+\hat{\alpha}$, and $\hat{v}_{i}$ is calculated from

$$
\hat{v}_{i}=t_{i}-\frac{\varphi_{i}}{1+\hat{\alpha}}-\left(t_{\text {ref }}-\frac{\varphi_{\text {ref }}}{1+\hat{\alpha}}\right) .
$$

With $\varphi_{i}$ from Equation (16), $\hat{v}_{i}$ can be expressed as

$$
\hat{v}_{i}=D_{i}-D_{r e f}-\frac{\alpha-\hat{\alpha}}{1+\hat{\alpha}}\left(t_{i}-t_{r e f}-\left(D_{i}-D_{r e f}\right)\right)
$$


The right term in Equation (18) represents the error of the estimate, $\hat{v}_{i}-v_{i}$. It is zero for $\hat{\alpha}=\alpha$.

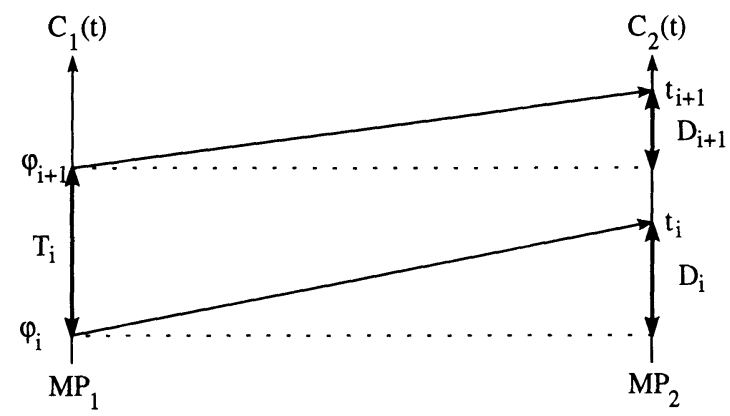

Figure 3 General configuration for CDV measurement.

For CBR traffic, 2-point CDV between the source and $\mathrm{MP}_{2}$ can be estimated on the basis of the arrival times of user cells, if the peak emission interval $T_{P}$ is known at $\mathrm{MP}_{2}$. In this case, $T_{i}=T_{P}$ (Figure 3 ) and $\hat{v}_{i}$ can be calculated with respect to a reference arrival time $\hat{c}_{i}$ by

$\hat{v}_{i}=t_{i}-\hat{c}_{i}$,

with the reference arrival time defined as

$\hat{c}_{0}=t_{0}, \hat{c}_{i+1}=\hat{c}_{i}+T_{P}$.

This definition corresponds to $D_{\text {ref }}=D_{0}$. However, it gives only correct results if no lost or misinserted cells occur during the measurement (ITU-T, 1993b). If there is a frequency offset between the clock in the source, from which $T_{P}$ is derived, and the clock $C_{2}$ at the measurement point, it has to be considered when estimating $v$. A frequency offset can be estimated with a regression analysis using the points $\left(\hat{c}_{i}, t_{i}-\hat{c}_{i}\right)$.

1-point CDV can be calculated on the basis of the cell arrival times $t_{i}$ only, according to the definition given by Equations (3) and (4). This requires $T_{P}$ to be known at the measurement point.

\section{EXAMPLE}

This section gives an example for the calculation of CTD and CDV according to the methods presented in the preceding sections. First, random numbers representing the delay were generated and added to the minimum delay $D_{\min }$. The queueing delay follows a gamma distribution $\Gamma(\gamma, \lambda)$ (Murakami, 1992). The sample included $n=1000$ cells (which corresponds to 2000 data points). Then the time stamps of $C_{2}$ were calculated (Equation (6)). The clock resolution was $1 \mu \mathrm{s}$. For the results presented here, the following values apply: $\gamma=4, \lambda=24, D_{\text {min }}=5000 \mu \mathrm{s}, \Delta T_{0}=5000 \mu \mathrm{s}, \alpha=2 \cdot 10^{-6}$ and $t_{u(i+1)}-t_{u i}=55000 \mu \mathrm{s}$. 

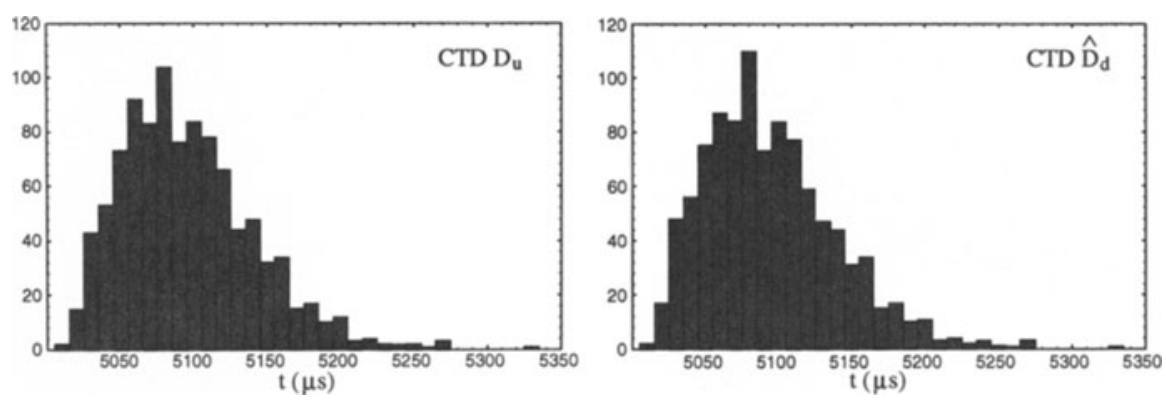

Figure 4 Frequency distribution of the actual delay $D_{u}$ and the estimated delay $\hat{D}_{u}$.

CTD was estimated as described in Section $4 . \hat{\alpha}$ and $\Delta \hat{T}_{0}$ were calculated using Equations (9) and (13) with $m=10$, and the estimate for the delay was determined by Equation (15). In this case, the frequency offset was estimated by $\hat{\alpha}=1.95 \cdot 10^{-6}$ and the time offset by $\Delta \hat{T}_{0}=5002.11 \mu \mathrm{s}$. Figure 4 shows the frequency distribution of the actual and the estimated delay in upstream direction. Table 1 compares mean, standard deviation, maximum and minimum of the actual and the estimated delay distribution. As the table shows, the estimated values are in good agreement with the actual values. The precision increases with the number of data points available.

Table 1 Mean, standard deviation, maximum and minimum of the actual and estimated delay

\begin{tabular}{ccccc}
\hline & Mean $(\mu \mathrm{s})$ & Standard dev. $(\mu \mathrm{s})$ & Maximum $(\mu \mathrm{s})$ & Minimum $(\mu \mathrm{s})$ \\
\hline$D_{u}$ & 5096.07 & 46.17 & 5355 & 5008 \\
$D_{d}$ & 5096.96 & 48.65 & 5281 & 5010 \\
$\hat{D}_{u}$ & 5094.91 & 46.22 & 5355 & 5007 \\
$\hat{D}_{d}$ & 5098.12 & 48.65 & 5283 & 5010 \\
\hline
\end{tabular}

2-point CDV was estimated as described in Section 5 from the same data. In the upstream direction, $C_{2}$ is the reference clock, and hence the frequency offset of $C_{1}$ relative to $C_{2}$ has opposite sign. Its estimate was $\hat{\alpha}=-2.10 \cdot 10^{-6}$. In downstream direction, the estimate for the frequency offset was $\hat{\alpha}=2.00 \cdot 10^{-6}$. Table 2 shows the estimated values for the 2-point CDV obtained by Equation (17).

Table 2 Mean, standard deviation, maximum and minimum of 2-point CDV

\begin{tabular}{lcccc}
\hline & Mean $(\mu \mathrm{s})$ & Standard dev. $(\mu \mathrm{s})$ & $\operatorname{Maximum}(\mu \mathrm{s})$ & $\operatorname{Minimum}(\mu \mathrm{s})$ \\
\hline$\hat{v}$ upstr. & 89.86 & 46.15 & 346.40 & 0 \\
$\hat{v}$ downstr. & 87.26 & 48.65 & 271.06 & 0 \\
\hline
\end{tabular}

Here, $D_{\text {ref }}=\operatorname{Min}\left\{v_{i}\right\}$ was selected. Then, assuming that $v_{i}=0$ corresponds to a cell with minimum delay $D_{\min }, \operatorname{Max}\left\{v_{i}\right\} / T_{C}$ can be interpreted as the maximum aggregate queue length, and $\bar{v} / T_{C}$ as the mean aggregate queue length of the cell queues within the 
connection. The frequency distribution of 2-point CDV is identical to the frequency distribution of CTD, shifted by $D_{\text {ref }}$.

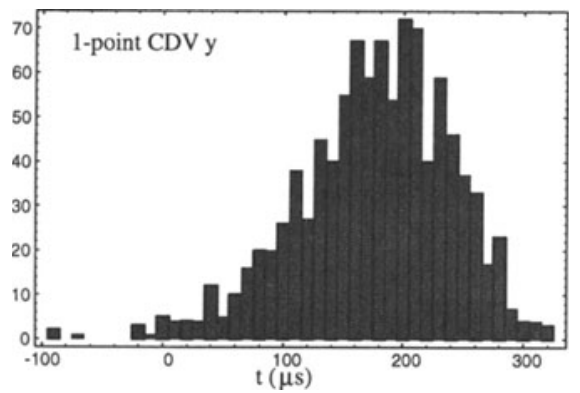

Figure 5 Frequency distribution of 1-point CDV $y$.

Table 3 Mean, standard deviation, maximum and minimum of 1-point CDV

\begin{tabular}{lcccc}
\hline & Mean $(\mu \mathrm{s})$ & Standard dev. $(\mu \mathrm{s})$ & Maximum $(\mu \mathrm{s})$ & Minimum $(\mu \mathrm{s})$ \\
\hline$y$ upstr. & 175.73 & 63.74 & 325.00 & -94.00 \\
$y$ upstr. $(\alpha=0)$ & 196.83 & 68.58 & 326.65 & -79.04 \\
\hline
\end{tabular}

1-point CDV was calculated from the data for the upstream direction, which corresponds to a CBR traffic whith $T_{P}=55000 \mu \mathrm{s}$. Figure 5 shows the frequency distribution of the $y_{i}$, and Table 3 gives characteristic values of the distribution. Note that there is a frequency offset between the clock in the source, from which $T_{P}$ is derived, and the clock $C_{2}$ at the measurement point. Since 1-point CDV includes the characteristics of the cell source, this is not considered here. Table 3 gives also the characteristic values of 1-point CDV for the case of $\alpha=0$. Figure 5 shows clearly the different shape of the 1-point CDV distribution compared with the CTD distribution of $D_{u}$ (and also the 2-point CDV distribution) in Figure 4, which is attributable to the definition of the reference arrival time as given by Equation (4).

\section{CONCLUSION}

This paper has discussed the origin and definition of CTD and the two parameters associated with CDV, 2-point CDV and 1-point CDV, and related estimation methods suited for inservice monitoring. The measurement of CTD and, in general, also of 2-point CDV requires the transmission of time-stamped cells. For in-service monitoring, this is done by means of performance management OAM cells. 1-point CDV, and in the case of CBR traffic also 2-point CDV, can be measured on the basis of the arrival times of user cells with respect to the peak cell rate $1 / T_{P}$.

The method presented for estimating CTD is based on a least-square linear regression analysis used to assess the frequency offset of the remote clock. The time offset is estimated proceeding on the assumption that some of the data points show a delay equal or close to the minimum delay, which is assumed to be equal in both the up- and downstream direction. This 
method could be implemented in a simple way if the loopback mechanism of OAM cells would support such delay measurements.

\section{REFERENCES}

Brinkmann, A., Macfadyen, R., Atkinson, A. and Scharf, E. M. (1994) Validation of delay and cell loss models for ATM switches. EXPLOIT traffic workshop, Basle, September 14-15.

Cuthbert, L. G. and Sapanel, J.-C. (1993) ATM - The broadband telecommunications solution. The Institution of Electrical Engineers, London.

Duda, A., Harrus, G., Haddad, Y. and Bernard, G. (1987) Estimating global time in distributed systems. Proceedings of the Conference on Distributed Computing Systems, Berlin.

Ellingson, C. E. and Kulpinski, R. J. (1973) Dissemination of system time. IEEE Transactions Communications, COM-21, 605-624.

Farkouh, S. C. (1993) Managing ATM-based broadband networks. IEEE Communications Magazine, May, 82-86.

Gruber, J. G. (1993) Performance and fault management functions for the maintenance of SONET/SDH and ATM transport networks. Proceedings ICC 93, Geneva.

ITU-T (1993a) Recommendation I.350: General aspects of quality of service and network performance in digital networks, including ISDNs. Geneva.

ITU-T (1993b) Recommendation I.356: B-ISDN ATM layer cell transfer performance. Geneva.

ITU-T (1993c) Recommendation I.371: Traffic control and congestion control in B-ISDN. Geneva.

ITU-T (1993d) Recommendation I.610: OAM principles of the B-ISDN access. Geneva.

Klepczynski, W. J. (1983) Modern navigation systems and their relation to timekeeping. Proceedings of the IEEE, 10, No. 10, 1193-1198.

Murakami, H., Sato, N. and Okamoto, T. (1992) Monitoring Method for Cell Transfer Performance in ATM Networks. NTT Review, 4, No. 4, 38-44.

\section{BIOGRAPHY}

Carsten Roppel is a researcher at the Research and Technology Centre of Deutsche Telekom since 1989. He studied Electrical Engineering at the Technical University of Darmstadt. Since then he has been involved in research projects in the field of ATM and interactive video services. In the framework of the European research program RACE he was involved in the project Technology for ATD. His research interests include high-speed access networks and customer premises networks and performance aspects of ATM systems. 